\title{
Huella de carbono en Latinoamérica como herramienta de medición de impacto ambiental en Instituciones privadas, 2017-2021
}

\author{
Nicanor Mellado Villafuerte \\ nimelladov@ucvvirtual.edu.pe \\ https://orcid.org/0000-0003-0312-1126 \\ Universidad César Vallejo \\ Cusco-Perú
}

Salinova Carrasco Huaman

scarrascoh@ucvvirtual.edu.pe

https://orcid.org/0000-0003-1844-4008

Universidad César Vallejo

\section{RESUMEN}

Cusco-Perú

La estimación de la huella de carbono es una herramienta importante en la medición de la emisión de GEI. En países de Latinoamérica la estimación tiene métodos variados y depende de las políticas climáticas implementadas por cada gobierno y de la iniciativa privada, por lo que no existe uniformidad de criterios entre los países ni dentro de ellos. Esto motiva la presente revisión que tiene como objetivos verificar las metodologías implementadas para la estimación de la huella de carbono en organizaciones privadas de Latinoamérica, los resultados de su huella de carbono y la importancia del uso de esta herramienta como parte de la acción frente al cambio climático; para ello se revisó artículos recopilados de la base de datos de repositorios importantes. La selección de artículos se realizó mediante el método Prisma. De los resultados se evidencia que el protocolo GHG y el propuesto por IPCC 20016 fueron las metodologías más usadas en la medición de la HC. Las actividades económicas de las organizaciones que midieron sus HC fueron diversas, que los consideraron como líneas de base para futuras comparaciones, sin embargo, no se encontraron estimaciones posteriores, lo que constituye un punto débil sobre todo si se trata de implementar y evaluar acciones de mejora. En esa línea las organizaciones propusieron medidas de mitigación de su HC como acciones de importancia para disminuir la emisión de GEI.

Palabras clave: ambiente; metodología; estimación; huella. 


\title{
Carbon footprint in Latin America as a tool for measuring environmental impact in private institutions, 2017-2021
}

\begin{abstract}
The estimation of the carbon footprint is an important tool in the measurement of GHG emissions. In Latin American countries, the estimation has varied methods and depends on the climate policies implemented by each government and the private initiative, so there is no uniformity of criteria between countries or within them. This motivates this review, which aims to verify the methodologies implemented to estimate the carbon footprint in private organizations in Latin America, the results of their carbon footprint and the importance of using this tool as part of actions against change climate; For this, articles collected from the database of important repositories were reviewed. The selection of articles was carried out using the Prisma method. From the results it is evident that the GHG protocol and the one proposed by IPCC 20016 were the most used methodologies in the measurement of $\mathrm{CH}$. The economic activities of the organizations that measured their $\mathrm{HC}$ were diverse, which considered them as baselines for future comparisons, however no subsequent estimates were found, which constitutes a weak point especially when it comes to implementing and evaluating improvement actions. . Along these lines, the organizations proposed mitigation measures for their $\mathrm{HC}$ as important actions to reduce GHG emissions
\end{abstract}

Keywords: atmosphere; methodology; estimate; paw print

Artículo recibido: 10 Setiembre. 2021 Aceptado para publicación: 15 Octubre. 2021 Correspondencia: nimelladov@ucvvirtual.edu.pe Conflictos de Interés: Ninguna que declarar 


\section{INTRODUCCIÓN}

La huella de carbono constituye una herramienta importante para medir el impacto ambiental que genera la Emisión de Gases de Efecto invernadero, GEI, en el cambio climático (Espindola \& Valderrama, 2018). Los GEI se producen como consecuencia de la actividad directa o indirecta de una empresa o de un individuo, en función a su estilo de vida o actividad económica, cuyo impacto en el medio ambiente dependerá de la cantidad de gases emitidos (Ministerio para la Transición Ecológica, 2018; Saavedra, 2020; Eckelman, 2018).

Con la firma del Acuerdo de Paris en el año 2015, donde casi 200 países se comprometieron a trabajar con el objetivo de mantener el aumento de la temperatura global por debajo de los $2^{\circ}$ centígrados, mediante un cambio estructural en las actividades productivas, económicas y de consumo, en forma sostenible y orientados a implementar políticas climáticas efectivas para alcanzar las metas respectivas (Cartes, 2021; Naciones Unidas, 2021).

Se ha estimado que los países desarrollados, de primer mundo, son lo que más contaminan debido a la mayor producción antropogénica de GEI, relacionado a sus actividades económicas y de mayor consumo. Sin embargo los mayores esfuerzos para mitigar la emisión de GEI, proviene de las empresas que de manera independiente o por las políticas climáticas que imponen el 'precio social de carbono, se ven sensibilizadas a dirigir sus esfuerzos, para lograr productos con menor huella de carbono a partir de sus procesos de producción (Cartes, 2021). En este contexto, países de primer mundo como Estados unidos y la Comunidad Europea ha tomado medidas de incentivo arancelario a las importaciones de productos en función a la emisión de GEI que generaron en el ciclo productivo.

La emisión de GEI puede ser generada por diversas actividades antropogénicas, desde la proveniente de la actividad estudiantil en campus universitarios debido al uso de transporte público (Yañez et al., 2020), pasando por actividades productivas como la lechera donde el mejor nivel de pastoreo estaba relacionado con mejores estándares ambientales (Palacios et al., 2021) y hasta las actividades prestacionales de salud de los hospitales, donde son más contaminantes cuanto más es su complejidad resolutiva (MacNeill et al., 2017; Karliner et al., 2019; Smith \& De-Titto, 2018). Obviamente las empresas industriales y de la actividad extractiva como la minera son por mucho las que 
contaminan más. La relación demográfica poblacional también es un factor directamente relacionada con mayor emisión de GEI en el mundo.

Producir, consumir, exportar o hacer cualquier actividad económica de producción y prestación de servicios, implica emisión de GEI, por lo que determinar la huella de carbono se hace importante para tomar las medidas respectivas que conduzcan a la mitigar o disminuir su emisión. En esta línea, el marketing ecológico representa una opción atractiva para las empresas, donde pueden mejorar sus negocios a partir de un mejor tratamiento de la huella de carbono en el proceso del ciclo de vida del producto (Chacón et al., 2016).

Los países más contaminantes en Latinoamérica fueron Brasil y México (Romero, 2020), en parte debido a su mayor densidad demográfica. En los países de Latinoamérica el uso de la huella de carbono como instrumento de ponderación, no ha sido armoniosa. La implementación de guías metodológicas para estimar la huella de carbono forman parte de las políticas climáticas de cada país; sin embargo no existe consenso en los métodos de medición, las mismas que varían de acuerdo a las establecidas por los gobiernos y por la iniciativa de la empresa privada, por lo que los registros de la huella de carbono, pueden variar, incluso dentro de un país y más aún entre los países de Latinoamérica (CEPAL, 2021). La obligatoriedad en la medición e información sobre la emisión de GEI de instituciones públicas o privadas y las acciones de mitigación en los procesos del ciclo de producción y de servicios, son motivos de éste análisis.

El problema se evidencia cuando las gestión ambiental debe ser liderada por el gobierno, a quien le corresponde unificar criterios de medición de huella de carbono, con un proceso de registros de las mediciones del sector público y privado, su evaluación respectiva y la implementación de medidas legales que conlleven a la corrección, mejoramiento o solución de las actividades generadoras de GEI con el fin de mitigarlos o en el mejor de los casos eliminarlos.

Para estimación de GEI se consideran dos tipos de emisión: directa, asociada a la fuente controlada o generadas por la organización, como es producida por ejemplo por el uso de calefacción y combustibles fósiles; indirecta cuya fuente es controlada por otra organización como es el caso del uso de energía eléctrica. Estos tipos de emisión se han agrupado en los denominados alcances que forman tres tipos: alcance1, que reúne las emisiones directas de la organización y los alcances 2 y 3 que reúne las emisiones 
indirectas (Secretaría Distrital de Ambiente, 2015; Ministerio para la Transición Ecológica, 2018). La determinación final se obtiene por la aplicación de fórmulas que se expresan en medidas métricas de toneladas de dióxido de carbono, $\mathrm{CO} 2$. El proceso de estimación de la huella de carbono también implica la ponderación de la emisión de GEI durante del ciclo de vida en la producción del bien, sobre todo en actividades económicas productivas.

El protocolo GHG (protocolo de Gases de Efecto invernadero), es una herramienta internacional de mayor uso para el cálculo de la Huella de carbono, $\mathrm{HC}$, es extensa y compleja pero contabiliza emisiones directas e indirectas de cualquier sector o actividad productiva, tomando en cuenta los 3 alcances considerados como fuentes de origen de la emisiones de GEI ((Ranganathan et al., 2005 ; Fong et al., 2014).

Un punto importante en la cuantificación de la huella de carbono, HC, es el que tienen que enfrentar los tomadores de decisión o gestores, debido a la complejidad y variada metodología en la determinación de los GEI. La heterogeneidad al respecto, no permite uniformizar los resultados y afectan la contabilidad de la emisión, auditoria y certificación, situación que menoscaba los esfuerzos tanto del estado como de las empresas en sus acciones de mejora ambiental y reducción de la emisión. Esta diversidad metodológica está lejos de ser resuelta sobre todo en países latinoamericanos, donde además existen factores colaterales asociados a la pobreza y el subdesarrollo.

Espíndola \& Valderrama (2016), consideran tres enfoques para definir y cuantificar la huella de carbono: el científico, el geopolítico y el empresarial. El científico abocado a la objetividad del cálculo y escalas de aplicación; el geopolítico a partir de las políticas ambientales, con el involucramiento de los países por acuerdos internacionales, están destinados a reducir de la emisión de GEI, sensibilizando u ordenado a las organizaciones para que informen sus HC y sus esfuerzos para lograr contener o mitigar el cambio climático; el empresarial, que toman la iniciativa y concretizan acciones pro ambientales en la reducción de sus huellas de carbono tomando en cuenta metodologías diversas convenientes para cada organización. El protocolo de Kioto, representa un esfuerzo internacional geopolítico con el compromiso mundial de reducir las emisiones (United Nation Climatic Change, 2021), establece que gases deben ser cuantificados por ser considerados contaminantes, como el dióxido de carbono, metano, óxido nitroso hasta el ultimadamente incorporado trifluoruro de nitrógeno. 
Como objetivos del presente estudio se plantea analizar las metodologías empleadas para el cálculo de la huella de carbono y los resultados de la estimación, en países de Latinoamérica, desarrolladas por organizaciones privadas; por otro lado evaluar la importancia del uso de la huella de carbono en estos países como herramienta para determinar la emisión de GEI y su consecuente impacto ambiental.

De esta manera el estudio permitirá observar con mayor claridad, como algunos sectores privados en países de Latinoamérica han desarrollado sus estrategias dirigidas a estimar la huella de carbono de sus organizaciones. En el caso el Perú la implementación como parte de la rectoría estatal ha sido deficiente, toda vez que recién desde el 2019 se ha creado la plataforma digital de registro de huella de carbono (MINAM, 2019) y la medidas para mitigar el impacto del efecto de los GEI no parecen ser trascendentes, además que hace poco, en noviembre de 2020 se emite la Resolución Ministerial N 237 2020-MINAM, documento que aprueba la Guía para el funcionamiento de la herramienta huella de carbono Perú con el fin de precisar los procedimientos para el registro de las mediciones respectivas de instituciones públicas y privadas (MIMAM, 2020).

Los gobiernos están en la obligación de implementar guías metodológicas que uniformicen los procesos de estimación de la $\mathrm{HC}$, cuyo alcance involucre tanto las organizaciones privadas y públicas. En este sentido la rectoría en materia climática debe ser liderada por la gestión pública. De esta forma, el estado puede medir el comportamiento de la $\mathrm{HC}$ en las organizaciones privadas y públicas, incentivándolas a realizar acciones de mitigación y compromisos eficientes de reducción de la emisión de GEI (González, 2020). En esta línea, España creó el Registro Nacional de Huella de Carbono, Compensación y Proyectos de Absorción de Dióxido de Carbono mediante el Real Decreto 163/2014, donde se evidenció que las organizaciones con mayor probabilidad de registrar sus huellas, pertenecieron al sector no manufacturero de reciente creación y con cultura ambiental transparente (Córdova et al., 2018).

\section{ESTRATEGIAS METODOLÓGICAS O MATERIALES Y MÉTODOS}

El presente artículo representa un estudio de revisión recabada de la base de datos de importantes repositorios que se detallan en la tabla 1. Los artículos a selección estuvieron comprendidos entre los años 2017 al 2021. EBSCO fue el repositorio que más artículos abordaron el criterio de búsqueda sobre la huella de carbono; en este sentido, de 217 estudios de EBSCO, diez cumplieron con los criterios para la presente revisión. 
Tabla 1. Artículos reportados según la base de datos de repositorios

\begin{tabular}{lcccccccc}
\hline Repositorio & Código & $\begin{array}{c}\text { Combinación de } \\
\text { búsqueda }\end{array}$ & Resultado & $\begin{array}{c}\text { Temática } \\
\text { (Estimación } \\
\text { de Huella de } \\
\text { Carbono) }\end{array}$ & Región & Acceso & Objetivos & $\begin{array}{c}\text { Artículos } \\
\text { seleccionados }\end{array}$ \\
\hline SCOPUS & 1 & huella de carbono & 13 & 3 & 3 & 3 & 1 & 1 \\
EBSCO & 2 & huella de carbono & 58 & 17 & 17 & 17 & 10 & 10 \\
PROQUEST & 3 & huella de carbono & 217 & 3 & 3 & 3 & 1 & 1 \\
SCIELO & 4 & huella de carbono & 50 & 3 & 3 & 3 & 2 & 2 \\
DIALNET & 5 & huella de carbono & 32 & 4 & 4 & 4 & 1 & 1 \\
Total & & & $\mathbf{3 7 0}$ & $\mathbf{3 0}$ & $\mathbf{3 0}$ & $\mathbf{3 0}$ & $\mathbf{1 5}$ & $\mathbf{1 5}$ \\
\hline
\end{tabular}

En resumen, en la tabla 1 se observa que de 370 artículos relacionados al tema de revisión, tomados de los repositorios señalados, 15 cumplen con los objetivos del estudio y al cual se tuvo acceso al texto completo en formato PDF.

Dentro de los criterios de inclusión se consideró aquellos estudios que se relacionen a organizaciones con estimación de la huella de carbono, los protocolos usados para ello, sus resultados, las consideraciones ambientales en la acciones de mejora, que se encuentren en un país latinoamericano y que se tenga accesos a la información en texto completo PDF.

Para la selección de los artículos se usó el método Prisma (Urrutia \& Bonfill, 2010) que fue adaptado para la presente revisión, diseñando un flujograma de acuerdo al método señalado, tal como se muestra en la figura 1.

Figura1. Flujograma para Selección de Artículos a Partir de Base de Datos

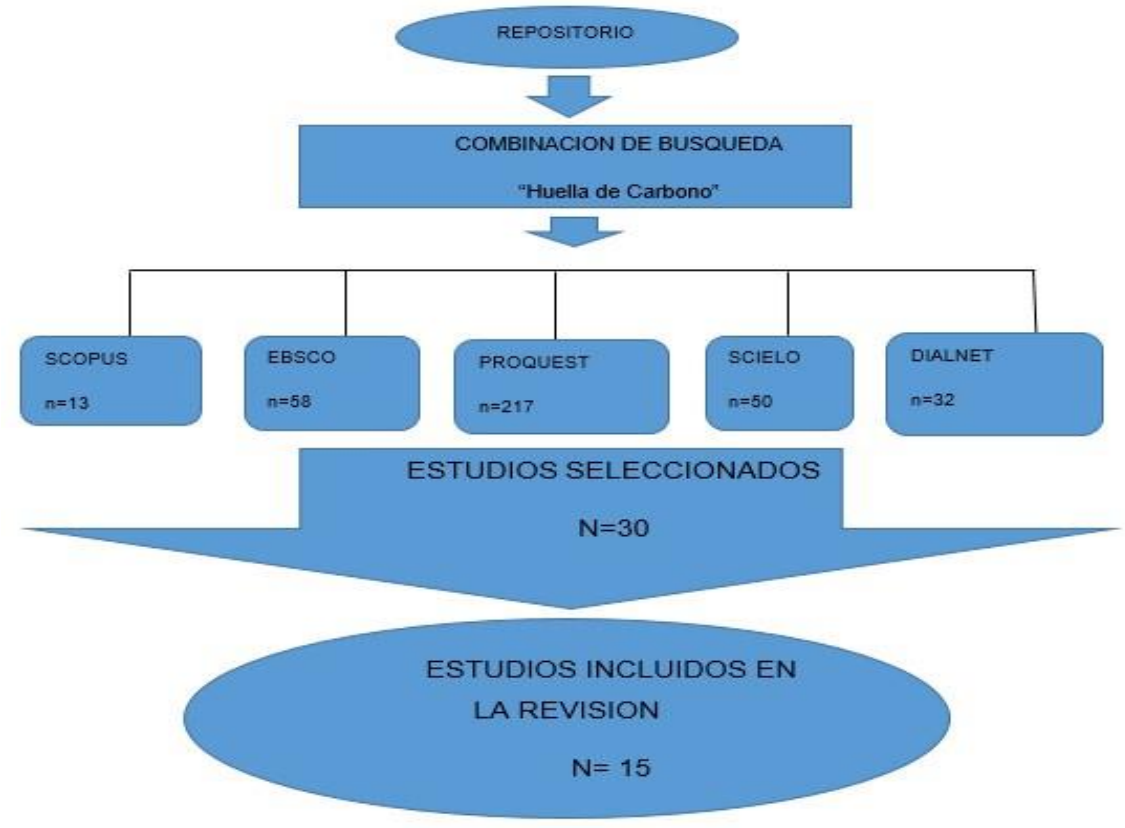

Nota. Flujograma para la selección de artículos que pasa por diferentes fases de la revisión sistémica. Adaptado de Declaración PRISMA: una propuesta para mejorar la publicación de revisiones sistemáticas y meta análisis (Urrutia \& Bonfill,

2010).

http://es.cochrane.org/sites/es.cochrane.o rg/files/public/uploads/PRISMA_Spanis $\underline{\text { h.pdf }}$ 


\section{RESULTADOS}

\section{Características de los Artículos}

Tal como se observa en la tabla 2, de la revisión de los artículos siguiendo el método Prisma, se observa que los estudios provienen de países diversos en su mayoría de Colombia, Perú y Ecuador, con tres artículos cada uno; México y Chile con dos, finalmente Cuba y Venezuela con uno. Los repositorios y los autores se detallan en la primera y segunda columna respectivamente.

\section{Tabla 2.}

Artículos seleccionados para revisión según repositorio, autor y país.

\begin{tabular}{lll}
\hline Repositorio & \multicolumn{1}{c}{ Autor } & \multicolumn{1}{c}{ País } \\
\hline EBSCO & (Canciano-fernández et al., 2020) & Cuba \\
EBSCO & (Guerra \& Rincón, 2018) & Venezuela \\
EBSCO & (Morales et al., 2018) & Perú \\
EBSCO & (Peniche et al., 2017) & México \\
EBSCO & (Bambarén Alatrista \& Alatrista Gutiérrez, & Perú \\
& 2016) & \\
\hline EBSCO & (Molina et al., 2018) & Colombia \\
EBSCO & (Jaime et al., 2018) & Colombia \\
EBSCO & (Salazar et al., 2019) & Ecuador \\
EBSCO & (Guallasamin \& Simón-Baile, 2018) & Ecuador \\
EBSCO & (Meza-lópez et al., 2021) & México \\
SCIELO & (Saavedra, 2020) & Perú \\
SCIELO & (Moreno et al., 2018) & Colombia \\
SCOPUS & (Balkenhol et al., 2018) & Chile \\
PROQUEST & (Yañez et al., 2020) & Chile \\
DIALNET & (Soto-Cabrera et al., 2020) & Ecuador \\
\hline
\end{tabular}

Los quince artículos realizaron estudios con enfoque cuantitativos, de los cuales trece presentaron un diseño exploratorio, uno correlacional y otro fue una revisión sistemática.

\section{Metodología utilizada para estimar la Huella de Carbono}

La metodología para estimar la HC es diversa y compleja, que depende de la actividad productiva o servicio que presta la organización. En este sentido el protocolo GHG trata 
de uniformizar y esquematizar la contabilidad de la emisión de GEI, que puede ser utilizado por cualquier tipo de organización, sea privada o pública, razón por la que se usó como punto de comparación con otros métodos. La tabla 3 muestra a las organizaciones y las actividades productivas que realizan, así como cuáles son los métodos usados para medir sus HC y los alcances considerados en la estimación. Así por ejemplo, una fábrica de vidrios en Cuba estima su HC utilizando la guía propuesta por el IPCC de año 2006 y el ISCC 205-2011, siendo el tipo de emisiones de GEI considerados como alcances 1 y 2. Según el protocolo GHG la estimación de la HC caracterizados por alcances, abarca las emisiones de GEI de acuerdo a las que generan las actividades directa o indirectamente, sea por consumo de energía o insumos, combustión de fósiles o tratamientos de desechos. La tabla 3 muestra en la tercera columna las fuentes de emisión de GEI agrupados en alcances o la considerada por la organización para efectos de medir la HC.

\section{Tabla 3.}

Métodos para estimación de HC de acuerdo a la organización, método de estimación y tipo de alcances considerados en la emisión de GEI.

\begin{tabular}{|c|c|c|}
\hline Organización o actividad productiva & Método de estimación & Alcances y fuentes de emisión \\
\hline $\begin{array}{l}\text { Fábrica de Vidrio de Cuba } \\
\text { (Canciano-fernández et al., 2020) }\end{array}$ & $\begin{array}{l}\text { IPCC-2006 (Panel Intergovernamental } \\
\text { de cambio Climático de 2006) } \\
\text { ISCC 205-2011 (International } \\
\text { Sustainability and Carbon Certification) }\end{array}$ & $\begin{array}{l}\text { Alcance } 1 \text { y } 2 \text {. Alcance } 1 \text { se cuantifico } \\
\text { emisiones asociadas a la descomposición } \\
\text { de los carbonatos y al horno de fusión de } \\
\text { vidrio. Alcance 2: al consumo de gas y } \\
\text { electricidad. }\end{array}$ \\
\hline $\begin{array}{l}\text { Campus Universitario de la } \\
\text { Universidad Central de Venezuela } \\
\text { (Guerra \& Rincón, 2018) }\end{array}$ & $\begin{array}{l}\text { Metodología de la Huella Ecológica - } \\
\text { HE- (Rees y Wackernagel, 1996) }\end{array}$ & $\begin{array}{l}\text { Alcances no definidos. HC de las } \\
\text { actividades del campus universitario y } \\
\text { sus componentes: área geográfica, } \\
\text { edificaciones, servicios y actividades de } \\
\text { la población universitaria. Por } \\
\text { universitario per cápita. }\end{array}$ \\
\hline $\begin{array}{l}100 \text { productores de Barranco y } \\
\text { Cañete en Lima. Fincas de maíz, } \\
\text { camote y yuca (Morales et al., 2018). }\end{array}$ & $\begin{array}{l}\text { La estimación se llevó a cabo con el } \\
\text { modelo de simulación programado en } \\
\text { Excel; Cool Farm Tool (CFT). }\end{array}$ & $\begin{array}{l}\text { Alcances no definidos. HC estimada de } \\
\text { la emisión por la producción y aplicación } \\
\text { de fertilizantes y plaguicidas. HC por el } \\
\text { uso energético de cultivos. HC por el } \\
\text { transporte de los productos. Por hectárea } \\
\text { de cultivo. }\end{array}$ \\
\hline $\begin{array}{l}\text { Vuelos internacionales de EE.UU a } \\
\text { México en el Puerto Vallarta (Peniche } \\
\text { et al., 2017) }\end{array}$ & $\begin{array}{l}\text { Calculadora de emisiones de la } \\
\text { Organización Internacional Civil de } \\
\text { Aviación (ICAO por sus siglas en inglés) }\end{array}$ & $\begin{array}{l}\text { Alcances no definidos. Estimación de la } \\
\text { HC de carbono por pasajero y HC total } \\
\text { en el } 2014 \text { en Puerto Vallarta. Considera: } \\
\text { origen de vuelo, destino, clase de asiento } \\
\text { y numero de pasajero. Combustible } \\
\text { utilizado. }\end{array}$ \\
\hline
\end{tabular}




\begin{tabular}{|c|c|}
\hline $\begin{array}{l}\text { HC en Cinco hospitales de tercer nivel } \\
\text { de Lima Perú. (Bambarén Alatrista \& } \\
\text { Alatrista Gutiérrez, 2016) }\end{array}$ & $\begin{array}{l}\text { Protocolo GHG y factores de emisión del } \\
\text { Ministerio de Energía y Minas de Perú }\end{array}$ \\
\hline $\begin{array}{l}\text { Ganadería Rumiantes en trópicos y } \\
\text { condiciones de pastoreo de Colombia } \\
\text { (Molina et al., 2018). }\end{array}$ & IPCC-2006 \\
\hline $\begin{array}{l}\text { Predios cafeteros en Colombia (Jaime } \\
\text { et al., 2018). }\end{array}$ & $\begin{array}{l}\text { Herramienta normativa NTC 5947, } \\
\text { específica para el análisis de remociones } \\
\text { y emisiones de GEI. Colombia }\end{array}$ \\
\hline
\end{tabular}

Campus de universidad San

Francisco de Quito. Ecuador (Salazar et al., 2019)
Diversa de acuerdo a la actividad que desarrolla la universidad. Para emisiones por consumo de energía y consumo de combustibles: método de Parra. Para residuos sólidos método de Peñafiel. Etc.

3 alcances. Alcance1: emisiones directas de calderos, grupos electrógenos y uso de móviles como ambulancias y otros vehículos. Alcance 2: Emisiones indirectas como adquisición y consumo de energía eléctrica. Alcance 3 generaciones de residuos sólidos y uso de agua potable.

No especifica alcances. Mide la fermentación entérica eliminada por los animales rumiantes.

No considera alcances. La determinación es sobre cantidad de combustible, energía, fertilizantes, fuentes de secado entre otros, hasta la entrega del café en el punto de venta.

3 alcances. Alcance 1: Consumo de diésel licuado de petróleo y el $\mathrm{CH} 4$ emitido de desechos orgánicos. Alcance 2: Emisiones indirectas a relacionada de energía comprada y consumida. Alcance 3: asociada a tratamiento de residuos sólidos, transporte y viajes aéreos de estudiantes, profesores y administrativos. Generación y tratamiento de aguas residuales.

3 alcances. 1: uso de combustibles de fuentes fijas y móviles, fertilizantes, etc. 2: uso de energía eléctrica 3: compra de combustibles, aceites, productos agrícolas, etc.

3 alcances: 1 consumo de combustibles fósiles móviles como el montacargas. 2: consumo de energía eléctrica. 3: otros como los fósiles vertidos mensualmente Alcance 2. Emisiones indirectas del consumo de energía eléctrica.

Cálculo basado en datos de la actividad multiplicado por el factor de emisión.
ambiental de la $u$
Universidad Nacional de Ingeniería.

Perú. (Saavedra, 2020)

Compañía agroindustrial de aceite de palma. Colombia (Moreno et al.,

Protocolo GHG e IPCC 2006.

Alcance 1. Relacionado a uso de 2018)

calderas, hornos y vehículos. No consideraron 2 y 3

3 alcances: 1. consumo de fuentes fijas como calderos y grupos electrógenos y

Hospital Base Puerto Montt. Chile (Balkenhol et al., 2018).
Protocolo GHG móviles por uso de vehículos. 2: consumo de energía eléctrica tomados de los resúmenes de facturas. 3: consumo de 


\begin{tabular}{|c|c|c|}
\hline & & $\begin{array}{l}\text { agua potable, uso de papel y generación } \\
\text { de residuos sólidos. }\end{array}$ \\
\hline Universidad de Talca. Chile & Protocolo GHG & $\begin{array}{l}3 \text { alcances. 1: combustible de GLP usado } \\
\text { para calefacción, transporte de personal } \\
\text { en el campus, transporte de estudiantes } \\
\text { en excursiones o practicas estudiantiles. } \\
\text { 2: adquisición y consumo de energía } \\
\text { eléctrica. 3: viajes aéreos y terrestres } \\
\text { usado por le personal y estudiantes, uso } \\
\text { de papel, desechos y vertederos. }\end{array}$ \\
\hline $\begin{array}{l}\text { Finca destilería en Ecuador. (Soto- } \\
\text { Cabrera et al., 2020) }\end{array}$ & $\begin{array}{l}\text { Herramienta Cool Farm Tool (CFT) e } \\
\text { IPCC. }\end{array}$ & $\begin{array}{l}\text { No alcances. Cuantifica el manejo de } \\
\text { residuos de cultivos. Uso de fertilizantes, } \\
\text { pesticidas, transporte fuera de la finca. }\end{array}$ \\
\hline
\end{tabular}

Como se aprecia la mayoría de los artículos muestran que los métodos de estimación de HC fueron heterogéneos y dependió de la actividad productiva o de los servicios que prestaron. Así mismo, sobre los alcances que agrupan las emisiones directas o indirectas fueron caracterizados en nueve estudios y en los restantes seis se basó en criterios de mensuración diseñados por sus metodologías. Las actividades productivas como es el caso de productores de maíz, camote y yuca en Lima, midieron sus HC sobre la el ciclo de producción de los productos, principalmente en la aplicación de fertilizantes y plaguicidas, uso de energía eléctrica y trasporte a destino final; situación parecida en el caso de producción de rosas de Ecuador donde caracterizaron sus fuentes de emisión por alcances.

Agrupar la emisiones en función a su origen permite delinear el concepto de alcances, categorización que no solo caracteriza la fuente de la emisiones sino que organiza mejor la contabilización y los reportes, además que trata de abarcar casi la totalidad de fuentes, de tal manera que la información sea más completa.

La tabla 4 resume las actividades de las organizaciones y sus protocolos. Se advierte que cinco utilizaron el protocolo GHG con actividades productivas diversas como la HC de la producción de rosas en una empresa privada de Ecuador, la HC de la producción de aceite de palma de Colombia, la HC del Hospital Puerto Montt de Chile, la HC del campus universitario de Talca-Chile y la HC de cinco hospitales de Lima-Perú. Esto permite observar que este protocolo es versátil y puede ser utilizado para la contabilidad de la HC de diversas actividades, tal como lo señala su guía respectiva (Fong et al., 2014). Otra metodología también utilizada en 4 organizaciones fue la propuesta por IPCC (Panel Intergubernamental de Cambio Climático), que el año 2006 diseñó las directrices para los 
inventarios nacionales de GEI (ipcc Intergovernmental Panel on Climate Change, 2006). En este contexto las actividades que midieron su $\mathrm{HC}$ fueron una fábrica de vidrios de Cuba, ganadería en trópicos de Colombia, Aserradero en México y cultivo de caña en Ecuador.

Las otras organizaciones restantes, también utilizaron otras metodologías y las actividades también fueron diversas.

\section{Tabla 4}

Protocolos o metodologías utilizadas en organizaciones productivas o de servicios.

\begin{tabular}{|c|c|c|c|}
\hline Protocolo & No & Organizaciones y periodo de estimación & País \\
\hline Protocolo GHG & 5 & 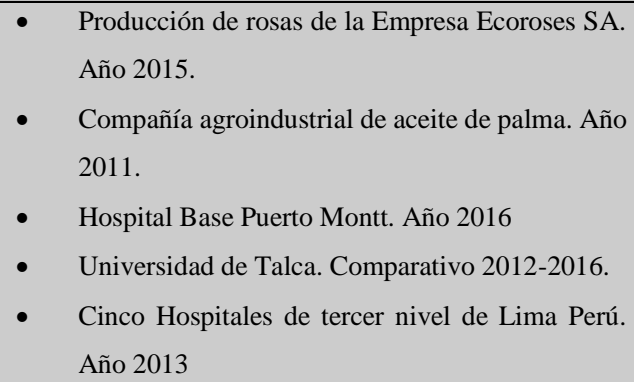 & $\begin{array}{l}\text { Chile } \\
\text { Chile }\end{array}$ \\
\hline IPCC 2006 & 4 & $\begin{array}{l}\text { - } \quad \text { Fábricas de vidrio de Cuba. Año } 2017 \\
\text { - } \quad \text { Ganadería de rumiantes en trópicos de Colombia. } \\
\text { Empresas Aserradero y fábrica de cajas Quintana, } \\
\text { y Sociedad de producción forestal El Diamante. } \\
\text { Periodo 2017-2018. } \\
\text { - Cultivo de caña de azúcar en la destilería Rivera } \\
\text { Revilla Fray Ángel. }\end{array}$ & $\begin{array}{c}\text { Cuba } \\
\text { Colombia }\end{array}$ \\
\hline $\begin{array}{l}\text { OTROS: Modelo de } \\
\text { simulación programado en } \\
\text { Excel; Cool Farm Tool } \\
\text { (CFT) } \\
\text { Metodología de la Huella } \\
\text { Ecológica, HE, de Rees y } \\
\text { Wackernagel. } \\
\text { Calculadora de emisiones } \\
\text { de la Organización } \\
\text { Internacional Civil de } \\
\text { Aviación. } \\
\text { Herramienta normativa } \\
\text { NTC } 5947 \text { de Colombia. }\end{array}$ & 6 & $\begin{array}{l}\text { - Campus universitario de la Universidad Central } \\
\text { de Venezuela. Periodo } 2011-2015 . \\
\text { Productores de Barranco y Cañete en Lima. } \\
\text { Fincas de maíz, camote y yuca. Periodo 2015- } \\
2016 . \\
\text { - } \quad \text { Vuelos internacionales de EE.UU a México en el } \\
\text { Puerto Vallarta. Año } 2014 \text {. } \\
\text { Predios cafeteros de Colombia. } \\
\text { Campus de universidad San Francisco de Quito. } \\
\text { Periodo } 2015 \text {. } \\
\text { Sistema de iluminaciones en cinco ambientes de } \\
\text { la facultad de Ingeniería Ambiental de la } \\
\text { universidad nacional de Ingeniería. Año } 2018 \text {. }\end{array}$ & $\begin{array}{c}\text { México } \\
\text { Colombia } \\
\text { Ecuador }\end{array}$ \\
\hline
\end{tabular}

Toda actividad antropogénica genera GEI en mayor o menor valor y su impacto en el cambio climático dependerá de la emisión contaminante y de las acciones de mitigación que emprende la organización; por tanto, medir su $\mathrm{HC}$ en forma periódica resulta ser 
necesaria para efectos de cambios de conducta, monitorización y mejoras amigables con el clima. La uniformización en las metodologías permite realizar comparaciones de la HC de actividades similares en distintas regiones de Latinoamérica y también de actividades diferentes para poder discriminar que actividades son más contaminantes y que organizaciones ejecutan acciones de mejora para mitigar sus HC. En este sentido según se observa en la tabla 4 la metodología usada para medir la HC fue diferente incluso dentro del mismo país, aspecto que refuerza la necesidad de que los gobiernos deban implementar guías metodologías únicas, que por un lado exijan a sectores privados y públicos el registro de sus huellas en plataformas digitales y por otro lado puedan monitorizar, incentivar y mitigar sus $\mathrm{HC}$.

La misma tabla evidencia como las estimaciones de la $\mathrm{HC}$ se han realizado en periodos anuales anteriores al 2018, desconociéndose si estas organizaciones actualizaron sus mediciones en años posteriores. Es una consideración importante, donde obliga la rectoría del estado en aspectos señalados en el párrafo anterior.

Siguiendo esta línea cabe resaltar que las estimaciones de $\mathrm{HC}$ en el campus universitario de la Universidad Central de Venezuela (Guerra \& Rincón, 2018) tuvieron mediciones en años distintos, lo que permitió advertir que un incremento de la contabilidad al 2017 fue resultado del incremento de estudiantes y personal docente y administrativo, observación que permite sugerir la necesidad de que las organizaciones deben medir sus HC periódicamente y contar con una línea de base de medición para crear un punto de partida a partir del cual puedan monitorizar sus HC y procurar medidas que conlleven a disminuir sus emisiones. En este sentido los demás estudios informaron sus HC como medida de base para posteriores cuantificaciones.

\section{RESULTADOS Y DISCUSIÓN}

\subsection{Resultados de la Huella de Carbono}

Los resultados de la medición de $\mathrm{HC}$ han variado de acuerdo al alcance medido o las fuentes de emisión según las actividades consideradas. La tabla 5 agrupa las organizaciones según actividades o servicios en: productivas, académicas, salud y otros, con los respectivos resultados más resaltantes de la $\mathrm{HC}$ considerados en porcentaje de emisión del total medido. Las fuentes de emisión de GEI tomadas para medición se detallan en la tabla 3. 
Se consideraron ocho organizaciones productivas donde se evidencia que la mayor emisión de GEI proviene del alcance 1 o de emisiones directas por el uso de insumos como fertilizantes y de combustibles fósiles de unidades fijas o móviles usados para el transporte. Por tanto se asume que las fuentes principales de emisión se generan durante el proceso del ciclo de vida del producto o los usados para la prestación de un servicio. En este sentido en la tabla 5 se observa que el 75\% de la emisión en el caso de fábrica de vidrio de Cuba proviene del uso de horno de fusión; el $83 \%$ de fuente de GEI resultó del uso de fertilizantes y transporte durante la producción de maíz, camote y yuca en Lima Perú; 98\% de la HC se estimó en la producción cafetera de Colombia derivado del uso sobre de fertilizantes nitrogenados, resultados también observados en el caso de producción de rosas en Ecuador y cultivo de caña de azúcar también en el Ecuador.

Un detalle importante resalta que la mayor fuente de emisión en caso de las universidades se encuentra en el alcance 3, como consecuencia del uso de transporte aéreo de estudiantes y docentes por motivos de capacitación o excursión; asimismo, por el uso de papel y el tratamiento de residuos sólidos. En el caso de actividades prestacionales de salud, las emisiones provienen de fuentes como los alcances 1 y 2 , que tienen que ver con el uso de combustibles fósiles de calderos, unidades móviles y consumo de energía eléctrica. Llama la atención que el manejo de residuos sólidos considerados como alcance 3 no son importantes en la $\mathrm{HC}$ de un establecimiento hospitalario, aunque esta afirmación no es significativa dado que se trata de 2 estudios seleccionados.

La importancia de medir la $\mathrm{HC}$ en toda actividad antropogénica radica en que ésta no se escapa de ser contaminante en mayor o menor grado. El uso de transporte aéreo es una fuente grande de emisión de GEI, no sólo por el uso de combustibles fósiles de los aviones sino por el uso de las edificaciones, insumos, alimentos, energía eléctrica, entre otros, presentes en las instalaciones aeroportuarias. El estudio de Peniche et al., (2017) sobre la HC de vuelos entre México y Estados Unidos muestra una significativa cifra de 210.850 t CO2eq por año, eliminadas al ambiente, que requiere 468.66 hectáreas de bosque para la captura de $\mathrm{C} 02$ que permitan mitigar las emisiones proveniente de esta actividad comercial. 


\section{Tabla 5.}

Resultado de la medición de HC según actividad o servicio realizado.

\begin{tabular}{|c|c|c|c|}
\hline Actividad & No & Organizaciones & Resultado de medición de la HC \\
\hline \multirow[t]{9}{*}{ Productiva } & 8 & Fábricas de vidrio de Cuba & $75 \%$ dentro del alcance 1 por el uso de horno de fusión. \\
\hline & & $\begin{array}{l}\text { Productores de Barranco y Cañete en Lima. } \\
\text { Fincas de maíz, camote y yuca }\end{array}$ & $\begin{array}{l}83 \% \text { de GEI provienen de aplicación de fertilizantes y } \\
\text { transporte }\end{array}$ \\
\hline & & Ganadería rumiantes en trópicos de & $60 \%$ proviene de la fermentación entérica de los ganados \\
\hline & & Colombia & \\
\hline & & Predios cafeteros de Colombia & $\begin{array}{l}98 \% \text { proviene del proceso de producción, cosecha y pos- } \\
\text { cosecha. Sobre todo relacionado al uso de fertilizantes } \\
\text { nitrogenados. }\end{array}$ \\
\hline & & Producción de rosas en Ecuador & $\begin{array}{l}68.26 \% \text { dentro del alcance } 1 \text { por el uso de fertilizantes, } \\
\text { fuentes fijas y móviles. }\end{array}$ \\
\hline & & Aserradero y fábrica de cajas en México & $\begin{array}{l}\text { La mayor huella provino del alcance } 1 \text { por el uso de } \\
\text { combustible para tractocamiones, montacargas y grúas. }\end{array}$ \\
\hline & & $\begin{array}{l}\text { Compañía agroindustrial de aceite de palma } \\
\text { en Colombia }\end{array}$ & $\begin{array}{l}61.05 \% \text { de emisión por tratamiento de aguas residuales } \\
\text { industriales. }\end{array}$ \\
\hline & & Cultivo de caña de azúcar en Ecuador & $\begin{array}{l}49.8 \% \text { de emisión por manejo de residuos de cultivo y } \\
\text { fertilizantes nitrogenados. }\end{array}$ \\
\hline \multirow[t]{4}{*}{ Académico } & 4 & $\begin{array}{l}\text { Campus universitario de la Universidad } \\
\text { Central de Venezuela }\end{array}$ & 43\% de emisión por la generación de residuos sólidos. \\
\hline & & $\begin{array}{l}\text { Campus de Universidad San Francisco de } \\
\text { Quito, Ecuador- }\end{array}$ & $\begin{array}{l}81.4 \% \text { en el alcance } 3 \text { debido al transporte de estudiantes, } \\
\text { profesores y administrativo en viajes aéreos. Tratamiento } \\
\text { de residuos sólidos. }\end{array}$ \\
\hline & & $\begin{array}{l}\text { Sistema de iluminación de en } 5 \text { ambientes } \\
\text { de la facultad de Ingeniería Ambiental de la } \\
\text { Universidad Nacional de Ingeniería, Perú }\end{array}$ & $\begin{array}{l}41.18 \% \text { de la emisión en le alcance } 2 \text { debido a la actividad } \\
\text { académica. }\end{array}$ \\
\hline & & Universidad de Talca, Chile & $\begin{array}{l}60 \% \text { en el alcance } 3 \text { por el transporte aéreo y terrestre de } \\
\text { estudiantes y docentes en excursiones. Uso de papel, } \\
\text { desechos y vertederos. }\end{array}$ \\
\hline \multirow[t]{2}{*}{ Salud } & 2 & 5 hospitales de tercer nivel de Lima Perú & $\begin{array}{l}46 \% \text { dentro del alcance } 1 \text { por el uso de calderos, grupos } \\
\text { electrógenos y móviles como ambulancias. }\end{array}$ \\
\hline & & Hospital Base Puerto Montt, Chile & $46 \%$ dentro del alcance 2 por el uso de energía eléctrica \\
\hline Otros & 1 & $\begin{array}{l}\text { Vuelos internacionales de EE.UU a México } \\
\text { en el Puerto Vallarta }\end{array}$ & 210.850 tCO2eq al año significativo para México. \\
\hline
\end{tabular}

Tal como se observa en la tabla 5, los resultados de las fuentes de emisión de GEI fueron diversos y dependieron del tipo de actividad o rubro al cual se dedican las empresas. Sin embargo, exceptuando al estudio de la $\mathrm{HC}$ de fábricas de Cuba cuya comparación con fábricas de España informan menor emisión de GEI (Canciano-Fernández et al., 2020), los demás reportes no comparan sus resultados con similares actividades en otros países del orbe. Esta situación lleva a observar que todavía existe un camino largo por mejorar 
y uniformizar las metodologías y los valores límites de HC referenciales al cual se debe alcanzar o sea deseable el que logre una organización sea pública o privada. En esta línea se debe recalcar el rol que deben jugar los gobiernos como entes rectores, no solo en el registro de $\mathrm{HC}$ a través de una plataforma digital o guías metodológicas, sino liderar el cambio de conductas y compromisos de mejora, con metas definidas e individualizadas para cada actividad o servicio.

Los estudios revisados, en forma unánime, consideran la importancia de la medición de la HC, que permitirá promover conductas de cambio y mejora orientados a disminuir la emisión de GEI, generados por sus organizaciones. En caso de actividades productivas como cultivos y producción de café, rosas, aceite de palma, caña de azúcar; entre otras acciones, estas organizaciones proponen, el uso racional de fertilizantes, mejora de técnicas de suelos y uso racional de combustible fósiles de unidades fijas y móviles. Sin embargo, estas sugerencias se han limitado a ser generales y no se trazaron líneas concretas para poder modificar los procesos, sea con el manejo de indicadores y metas como unidades de medida a ser tomados en cuenta para el proceso de cambio. Sin embargo, hay que resaltar cómo la iniciativa privada en Latinoamérica lidera sus acciones frentes al cambio climático, en procura de mitigar sus emisiones de GEI a través de sus estimaciones de $\mathrm{HC}$, esfuerzo que todavía requiere la mayor participación del estado con políticas publicas ambientales más contundentes y eficaces.

\section{CONCLUSIÓN O CONSIDERACIONES FINALES}

La estimación HC constituye una herramienta importante para medir el grado de impacto que genera la actividad antropogénica en el cambio climático. Existe un esfuerzo por parte de organizaciones privadas por medir sus HC, las mismas que recurrieron a metodologías diferentes para la ponderación de sus emisiones, según como lo vieron pertinentes, adaptando un diseño de acuerdo a sus actividades económicas o de servicios. La presente revisión seleccionó 15 artículos que involucraron organizaciones que midieron sus HC y los resultados fueron diversos y de acuerdo a la actividad que realizaron.

La mayoría de empresas utilizaron el protocolo GHG para estimar sus HC, guía que agrupa las fuentes de emisiones de GEI en los denominados alcances, concepto que permite abarcar la mayor parte de actividades antropogénicas que desarrolla la organización para la producción de bienes o servicios. Un punto fuerte de este protocolo es su versatilidad, debido a que puede ser usado en cualquier actividad. El alcance 1 se reportó como mayor resultado de HC y que tiene que ver con las emisiones directas 
propias del desarrollo productivo de la institución. El protocolo IPCC 2006, fue el segundo más utilizado y también fue empleado por diferentes instituciones con distintos rubros.

La estimación de HC, reportó que las organizaciones son generadoras de contaminación y de emisión de GEI independiente de la actividad que desarrollan, no obstante al no contar con patrones de comparación de $\mathrm{HC}$ con valores límite de emisión deseable menoscaban el análisis y llevan a deducir probablemente en forma errónea cuales actividades son más o menos contaminantes.

Las acciones de mitigación reportadas según la revisión no han sido concretas y se basaron en generalidades, sugiriendo cambio de conductas y mejora en los procesos de producción y de servicios. EL uso de indicadores resultaría una herramienta importante en la medición y trazado de metas que la organización y los organismos involucrados en el tema, deberían implementar en sus procesos de mejora.

\section{LISTA DE REFERENCIAS}

Balkenhol, M., Castillo, A., Soto, M., Feijoo, M., \& Merino, W. (2018). Huella de carbono en el Hospital Base de Puerto Montt. Revista Medica de Chile, 146, 13841389. https://doi.org/http://dx.doi.org/10.4067/s0034-98872018001201384

Bambarén Alatrista, C., \& Alatrista Gutiérrez, M. del S. (2016). Huella de carbono en cinco establecimientos de salud del tercer nivel de atención de Perú, 2013. In Revista Peruana de Medicina Experimental y Salud Pública (Vol. 33, Issue 2, p. 274). https://doi.org/10.17843/rpmesp.2016.332.2141

Canciano-fernández, J., Reinosa-Valladares, M., Hernández-Garcés, A., NúñezHernández, M., \& Ramírez-Díaz, L. (2020). Estimación de la huella de carbono en la producción de vidrio en Cuba. Minería y Geología, 36(4), 428-438. http://web.a.ebscohost.com/ehost/pdfviewer/pdfviewer?vid=22\&sid=7f0e3d 91 06d4-4c57-b2e5-628b89c3055c\%40sdc-v-sessmgr03

Cartes, F. (2021). Metodología para la estimación del precio social del carbono en Chile y los países de América Latina y el Caribe Gracias por su interés en esta publicación de la CEPAL. CEPAL. https://repositorio.cepal.org/bitstream/handle/11362/46957/1/S2100324_es.pdf

CEPAL. (2021). Huella Ambiental y de Carbono en las Exportaciones y el Comercio Internacional. Biblioteca de La CEPAL. 
https://biblioguias.cepal.org/c.php?g=587382\&p=4073966

Chacón, I., Pinzón, A., Ortegón, L., \& Rojas, P. (2016). Alcance y gestión de la huella de carbono como elemento dinamizador del branding por parte de empresas que implementan estas prácticas ambientales en Colombia \&. 32, 278-289. http://web.a.ebscohost.com/ehost/pdfviewer/pdfviewer?vid=14\&sid=7f0e3d9106d4-4c57-b2e5-628b89c3055c\%40sdc-v-sessmgr03

Córdova, C., Zorio-Grima, A., \& García-Benau, M. (2018). Nuevas Formas De Reporting Corporativo: Información Sobre La Huella De Carbono En España. Revista de Administração de Empresas, 58(6), 537-550. https://doi.org/10.1590/s0034759020180603

Eckelman. (2018). The US Health Care Sector's Carbon Footprint: Stomping or Treading Lightly? 108(52), 556-557. https://doi.org/10.2105/AJPH.2017.304160

Espindola, C., \& Valderrama, J. (2018). Huella del carbono Cambio Climático, Gestión Sustentable y Eficiencia Energética (Editorial Universidad de la Serena (ed.); Primera ed). https://web.kaizen-certification.com/wpcontent/uploads/2013/03/Libro-Huella-de-Carbono-dedicado-amigos-de-kaizen.pdf Espíndola, C., \& Valderrama, J. (2016). AbaniCO 2 : Un Método Simple y Efectivo para la Toma de Decisiones sobre la Adopción de la Huella del Carbono en la Gestión Sustentable de Emisiones en las Empresas. 27(3), 35-52. https://doi.org/10.4067/S0718-07642016000300005

Fong, W., Sotos, M., Doust, M., Schultz, S., Marques, A., \& Deng-Beck, C. (2014). Protocolo global para inventarios de emisión de gases de efecto invernadero a escala comunitaria. Word Resources Institute. https://ghgprotocol.org/sites/default/files/standards/GHGP_GPC \%28Spanish\%29.pdf

González, K. (2020). Medición y neutralización de la huella de carbono : evaluación al lento despegue de las iniciativas en Chile. La Tercera, 4-7. https://www.proquest.com/newspapers/medición-y-neutralización-de-la-huellacarbono/docview/2351384825/se-2?accountid=37408

Guallasamin, K., \& Simón-Baile, D. (2018). Huella de carbono del cultivo de rosas en Ecuador comparando dos metodologías: GHG Protocol vs . PAS 2050. Revista Latinoamericana de Estudios Socioambientales, 24, 27-56. 
https://doi.org/https://doi.org/10.17141/letrasverdes.24.2018.3091

Guerra, J., \& Rincón, I. (2018). Cálculo de la Huella Ecológica Campus de la Universidad Central de Venezuela. Luna Azul, 46, 3-20. https://doi.org/10.17151/luaz.2018.46.2 ipcc Intergovernmental Panel on Climate Change. (2006). 2006 IPCC Guidelines for National Greenhouse Gas Inventories. https://www.ipccnggip.iges.or.jp/public/2006gl/spanish/index.html

Jaime, J., Hernández, A., Miguel, N., Jairo, H., Suarez, P., Andrade, J., Andrea, M., Cano, V., Patricia, Y., Cortes, M., Fernando, J., \& Chavez, C. (2018). Determinación de la huella de carbono en el sistema de producción de café pergamino seco de cuatro municipios del sur del departamento del Huila ( Colombia ). Revista de Investigación Agraria $y$ Ambiental,

9. http://web.a.ebscohost.com/ehost/pdfviewer/pdfviewer?vid=17\&sid=7f0e3d9106d4-4c57-b2e5-628b89c3055c\%40sdc-v-sessmgr03

Karliner, J., Slotterback, S., R., B., Ashby, B., \& Steele, K. (2019). Huella Climatica del Sector de la $\quad$ salud. https://media.businesshumanrights.org/media/documents/files/documents/Huella_climática_del_sector_sa lud.pdf

MacNeill, A., Lillywhite, R., \& Brown, C. (2017). The impact of surgery on global climate: a carbon footprinting study of operating theatres in three health systems. The Lancet Planetary Health, 1(9), e360-e367. https://doi.org/10.1016/S25425196(17)30162-6

Meza-lópez, P., Trujillo-delgado, M., Burciaga-álvarez, A., Cruz-carrera, R., \& Nájeraluna, J. (2021). Carbon footprint estimate in the primary wood processing industry in El Salto , Durango Estimación de la huella de carbono en la industria de transformación primaria de la madera en El Salto, Durango. Revista Chapingo Serie Ciencias Forestales y Del Ambiente, 27, 127-142. https://doi.org/10.5154/r.rchscfa.2019.07.060

MIMAM. (2020). Guia para el funcionamiento de la herramienta huella de carbono Perú. https://www.gob.pe/institucion/minam/normas-legales/1364262-237-2020minam

MINAM. (2019). MINAM presenta plataforma digital "Huella de Carbono Perú." https://www.gob.pe/institucion/minam/noticias/69318-minam-presenta-plataforma- 
digital-huella-de-carbono-peru

Ministerio para la Transición Ecológica. (2018). Guía para el cálculo de la huella de carbono y para la elaboración de un plan de mejora de una organización. Gobierno de España, 52. https://www.miteco.gob.es/es/cambio-climatico/temas/mitigacionpoliticas-y-medidas/guia_huella_carbono_tcm30-479093.pdf

Molina, R., Sánchez, H., \& Mateus, D. (2018). Livestock Greenhouse Gases Emissions Under Grazing Conditions in the TropicLivestock Greenhouse Gases Emissions Under Grazing Conditions in the Tropic. Revista de Investigación Agraria $y$ Ambiental, 10(1), 91-106. https://doi.org/10.22490/21456453.2685

Morales, R., Zorogatúa, P., De Mendiburu, F., \& Quiroz, R. (2018). Producción Mecanizada de Maíz, Camote y Yuca en la Costa Desértica Peruana: Estimación de la Huella de Carbono y Propuestas de Mitigación. Ecología Aplicada, 17(1). https://doi.org/http://dx.doi.org/10.21704/rea.v17i1.1169

Moreno, J., Martínez, L., Belalcázar, L., \& Yecid, N. (2018). Corporate Carbon Footprint of a Palm Oil Mill. TECCIENCIA, 13(24), 1-10. https://doi.org/https://doi.org/10.18180/tecciencia.2018.24.1

Naciones Unidas. (2021). Objetivos de Desarrollo Sotenible. https://www.un.org/sustainabledevelopment/es/development-agenda/

Palacios, C., Plaza, J., Revilla, I., Nieto, J., Hidalgo, C., \& Sánchez, M. (2021). Milk Quality and Carbon Footprint Indicators of Dairy Sheep Management Systems. Animals, 11(5). https://doi.org/DOI: 10.3390 / ani11051426

Peniche, S., Laure, A., \& Cázares, L. (2017). El impacto ambiental del turismo internacional : caso de la huella de carbono de los vuelos internacionales hacia Puerto Vallarta, Jalisco, México. Revista de Investigaciones Turísticasurísticas, 14, 45-62. https://doi.org/. https://dx.doi.org/10.14461/INTURI2017.14.03

Ranganathan, J., Murphy, A., Eaton, R., \& Mcmahon, M. (2005). Protocolo de Gases de Efecto Invernadero. In World Business Council fr Sustainable Developent Secretaria de Medio Ambiente y Recursos Naturales. https://ghgprotocol.org/sites/default/files/standards/protocolo_spanish.pdf

Romero, E. (2020). ¿Qué es la huella de carbono y cuáles son los países con más emisiones? Elements. https://www.elementsgroup.com.ec/que-es-la-huella-decarbono-y-cuales-son-los-paises-con-mas-emisiones/ 
Saavedra, E. (2020). Huella de carbono- emisiones de GEI por uso del sistema de iluminación de la facultad de ingeniería ambiental de la universidad nacional de ingeniería, $\quad$ Lima-Perú. $\quad$ Scielo, 30(1), 121-138. https://doi.org/http://dx.doi.org/10.21754/tecnia.v30i1.827

Salazar, F., Valencia, M., Velasco, A., \& Ochoa-herrera, V. (2019). Actualización de la huella de carbono de la Universidad San Francisco de Quito USFQ para el año 2015. Avances En Ciencias e Ingenierías, 11(18), 254-265. https://doi.org/http://dx.doi.org/10.18272/aci.v11i2.450 255

Secretaría Distrital de Ambiente. (2015). Guía para el cálculo y reporte de Huella de Carbono Corporativa. Alcaldia Mayor de Bogotá. http://www.ambientebogota.gov.co/en/c/document_library/get_file?uuid=f64a7ccd8a76-4d0d-b6de-33a3f08576fc\& groupId=586236

Smith, M., \& De-Titto, E. (2018). Hospitales sostenibles frente al cambio climático: huella de. Revista Argentina de Salud Publica, 9(36), 7-13. http://rasp.msal.gov.ar/rasp/articulos/volumen36/7-13.pdf

Soto-Cabrera, A., Panimboza-Ojeda, A., Ramones-Pinargote, A., Pérez-Martínez, A., Sarduy-Pereira, L., \& Diéguez-Santana, K. (2020). Huella de Carbono en el Cultivo de la Caña de Azucar. Evaluación Agrícola de un Caso de Estudio de la Amazonía $\begin{array}{lll}\text { Ecuatoriana. Ingenio Magno, } & \text { 11(1). }\end{array}$ https://dialnet.unirioja.es/servlet/articulo?codigo=7537791

United Nation Climatic Change. (2021). ¿Qué es el Protocolo de Kyoto? https://unfccc.int/es/kyoto_protocol

Urrutia, G., \& Bonfill, X. (2010). Declaracion PRISMA: una propuesta para mejorar la publicación de revisiones sistemáticas y metaanálisis. Medicina Clínica, 135(11), $507-511$.

http://es.cochrane.org/sites/es.cochrane.org/files/public/uploads/PRISMA_Spanish. pdf

Yañez, P., Sinha, A., \& Marcia, V. (2020). Carbon Footprint Estimation in a University Campus: Evaluation and Insights. Sustainability, 12(1), 1-16. https://doi.org/DOI:10.3390/su12010181 